\title{
Thermodynamic loss mechanisms and strategies for efficient
}

\section{hot-electron photoconversion}

Cheng Zhang, ,ab Guoyang Cao, ${ }^{\text {a,b }}$ Shaolong Wu, ${ }^{\text {a,b }}$ Weijia Shao, ${ }^{\text {a,b }}$ Vincenzo

Giannini, ${ }^{c, d}$ Stefan A. Maier, ${ }^{c, e}$ and Xiaofeng $\mathrm{Li}^{\mathrm{a}, \mathrm{b},}$,

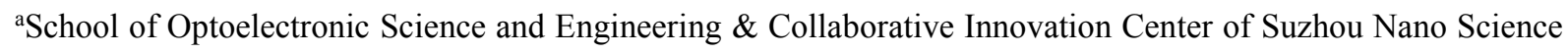
and Technology, Soochow University, Suzhou 215006, China;

${ }^{\mathrm{b}}$ Key Lab of Advanced Optical Manufacturing Technologies of Jiangsu Province \& Key Lab of Modern Optical Technologies of Education Ministry of China, Soochow University, Suzhou 215006, China;

'Blackett Laboratory, Imperial College London, Prince Consort Road, London SW7 2BZ, United Kingdom;

${ }^{\mathrm{d}}$ Instituto de Estructura de la Materia (IEM-CSIC), Consejo Superior de Investigaciones Cientificas, Serrano 121, 28006 Madrid, Spain;

${ }^{e}$ Chair in Hybrid Nanosystems, Nanoinstitute Munich, Faculty of Physics, Ludwig-Maximilians-Universität München, 80799 München, Germany.

${ }^{*}$ Corresponding author: xfli@suda.edu.cn

\section{S1. Hot-electron generation calculation}

The optical model aims at calculating the optical response and hot electron generate rate in the device. Based on the refractive index from Palik [S1], the finite element method (FEM) simulations are conducted using Comsol to solve Maxwell's equations [S2]. The periodic boundary conditions on the sides, perfect match layers for the top and bottom layers, and scattering boundary conditions for the top and bottom boundaries are set. The optical absorption efficiency $(A)$ by in the metal layers is calculated using [S3]:

$$
A(\omega)=\frac{\oiiint_{V} Q_{\mathrm{rh}}(x, y, z, \omega) d V}{P_{\text {in }}}
$$

where $\omega$ is the angular frequency, $P_{\text {in }}$ is the incident power, and $Q_{\text {rh }}$ is the local Ohmic loss in the metal as a function of the local electric field intensity:

$$
Q_{\mathrm{rh}}(x, y, z, \omega)=\frac{1}{2} \varepsilon_{i} \omega|E(x, y, z, \omega)|^{2}
$$

where $\varepsilon_{\mathrm{i}}$ is the imaginary part of the material permittivity and $E(x, y, z)$ the electric field at position $(x, y, z)$. For the low photon energy considered here, the higher-order hot electron transition processes such as multiple electron-hole pairs or multiple photons are forbidden, so 
one absorbed photon generates only one hot electron-hole pair [S4]. Taking into account the wavelength dependent resistive loss $\left(\eta_{\mathrm{res}}\right)$ of the absorbed energy, which is dissipated without the generation of the hot electrons, the local generation rate $(G)$ can be obtained:

$$
G(x, y, z, \omega)=\eta_{e h} Q_{\mathrm{rh}}(x, y, z, \omega) /(\hbar \omega)=\eta_{e h} \varepsilon_{i}|E(z, \omega)|^{2} /(2 \hbar)
$$

where $\hbar$ is the reduced Planck constant and $\eta_{\mathrm{eh}}=1-\eta_{\mathrm{res}}$ is the efficiency of plasmonic decay into hot electrons.

\section{S2. Energy distribution and flux of hot electrons reaching the $M / S$ interface}

The initial hot-electron energy distribution $D(E)$ is described by the simplified expression of the electron distribution joint density of states and normalized as [S5-S8]:

$$
D(E)=\frac{\rho(E-h v) f(E-h v) \rho(E)[1-f(E)]}{\int_{0}^{\infty} \rho(E-h v) f(E-h v) \rho(E)[1-f(E)] d E}
$$

where $E$ is the excited hot electron energy, $h v$ the photon energy, $\rho(x)$ the parabolic electron density of states at energy level of $x$, and $f(x)$ the corresponding Fermi distribution function at temperature $T=300 \mathrm{~K}$. Based on the assumption of an isotropic momentum distribution and using the exponential attenuation model, the probability of an electron with energy $E$ arriving at the $\mathrm{M} / \mathrm{S}$ interface under the diffusing angle $\theta$ is evaluated by [S9]:

$$
P_{e t r}(E, \theta, z)=\left\{\begin{array}{lc}
\frac{1}{2} \exp \left(-\frac{d(z)}{l_{M F P}(E) \cos \theta}\right), & \text { if }-\frac{\pi}{2}<\theta<\frac{\pi}{2} \\
0, & \text { otherwise }
\end{array}\right.
$$

where $d$ is the distance from the hot-electron generation position to the M/S interface and $l_{\mathrm{MFP}}$ the energy-dependent mean-free path of hot electrons accounting for electron-electron and electron-phonon contributions in Au. The transport efficiency ( $\eta_{\text {etr }}$ in the main text) of hot electrons with excess energy $E$ to reach Schottky interface is linked by $P_{\text {etr: }}$ :

$$
\eta_{e t r}(E)=\frac{\int_{0}^{d} A u \int_{-\frac{\pi}{2}}^{\frac{\pi}{2}} G(z) \times D(E) \times P_{e t r}(E, \theta, z) d \theta d z}{\int_{0}^{d} A u(z) \times D(E) d z}
$$

The flux of electrons $N_{\text {int }}(E, \theta)$ with excess energy $E$ to reach the interface under an angle $\theta$ is:

$$
N_{\text {int }}(E, \theta)=\int_{0}^{d_{\mathrm{Au}}} G(z) \times D(E) \times P_{e t r}(E, \theta, z) d z
$$

Then, the overall transport efficiency $\eta_{\text {trans }}$ is defined as the fraction of the electron flux arrived at the interface to all generated electrons:

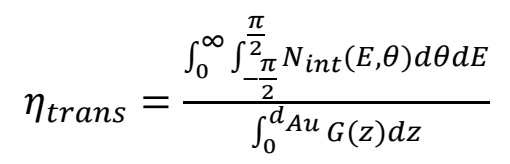

\section{S3. Injection efficiency of hot electrons into semiconductor}

With a simple, parabolic free-electron dispersion relation for the electrons and a well-defined 
effective mass, the kinetic energy of electrons in metal $\left(E_{k, m}\right)$ is:

$$
E_{k, m}=\frac{\hbar^{2}}{2 m_{e, A u}^{*}}\left(k_{A u}^{2}\right)
$$

The kinetic energy of electrons upon entering the semiconductor $\left(E_{k, s}\right)$ is:

$$
E_{k, s i}=\frac{\hbar^{2}}{2 m_{e, s}^{*}}\left(k_{s i}^{2}\right)
$$

where $m_{e, A u}^{*}\left(m_{e, s i}^{*}\right)$ is the effective mass of electrons in $\mathrm{Au}(\mathrm{Si})$ and $k_{A u}\left(k_{s i}\right)$ the hot electron momentum of electrons in $\mathrm{Au}(\mathrm{Si})$.

For electrons to inject into the semiconductor, the kinetic energy corresponding to the normal electron component of the electron momentum should be larger than the Schottky barrier, which defines an escape cone of angle $\Omega_{1}[\mathrm{~S} 10]$ :

$$
\begin{gathered}
E_{k, m} \cos \left(\Omega_{1}\right)=\frac{\hbar^{2}}{2 m_{e, A u}^{*}}\left[k_{A u} \cos (\Omega)\right]^{2}=\varphi_{S B} \\
k_{A u} \cos \left(\Omega_{1}\right)=\sqrt{2 m_{e, A u}^{*} \varphi_{S B}} / \hbar
\end{gathered}
$$

Moreover, for the translation invariance of the planar M/S interface, the momentum component parallel to the interface $\left(k_{\mathrm{y}}\right)$ is conserved during the charge transfer process. The continuity of the $k_{\mathrm{y}}$ sets a limit of the possible momentum parallel to the interface in the Au side [S11]:

$$
k_{A u} \sin \left(\Omega_{2}\right)=k_{s i}
$$

The escape angle $\Omega$ is the minimum of $\Omega_{1}$ and $\Omega_{2}$.

With the consideration of the highly anisotropic distribution of electron momenta at the interface, the fraction of electrons with sufficient magnitude of kinetic energy corresponding to normal components of the momentum determines the emission probability:

$$
\eta_{e s c}(E)=\frac{\int_{0}^{\Omega} 2 \pi \sin \theta d \theta \times N_{i n t}(E, \theta)}{\int_{0}^{\frac{\pi}{2}} 2 \pi \sin \theta d \theta \times N_{i n t}(E, \theta)}
$$

To more accurately calculate the injection efficiency, the possible reflection at the $\mathrm{M} / \mathrm{S}$ interface arising from the momentum mismatch in both media has to be considered. The transmission probability under the diffusing angle $\theta$ across the interface is given by [S11]:

$$
T=\frac{4 \sqrt{\left(k_{A u}^{2}-k_{y}^{2}\right)\left(k_{s}^{2}-k_{y}^{2}\right)}}{\left(\sqrt{k_{A u}^{2}-k_{y}^{2}}+\sqrt{k_{s i}^{2}-k_{y}^{2}}\right)^{2}}
$$

where $k_{y}=k_{A u} \sin (\theta)$. Then the injection efficiency is obtained as:

$$
\eta_{i n j}(E)=\frac{\int_{0}^{\Omega} 2 \pi \sin \theta d \theta \times N_{i n t}(E, \theta) \times T}{\int_{0}^{\frac{\pi}{2}} 2 \pi \sin \theta d \theta \times N_{i n t}(E, \theta)}
$$

It is noted that the upper limits of the integral in the denominators of Equation S14 and Equation S16 is $\pi / 2$ due to the fact that all the electrons arrived at the interface are moving 
forward to the M/S interface, so only half space is considered. The energy distribution $F(E)$ of the collected hot electrons is:

$$
F(E)=\frac{\int_{-\frac{\pi}{2}}^{\frac{\pi}{2}} N_{i n t}(E, \theta) d \theta \times \eta_{i n j}(E)}{\int_{\varphi_{S B}}^{\infty} \int_{-\frac{\pi}{2}}^{\frac{\pi}{2}} N_{i n t}(E, \theta) d \theta \times \eta_{i n j}(E) d E}
$$

Finally, the photocurrent density can be expressed as:

$$
J=e \int_{\varphi_{S B}}^{\infty} \int_{-\frac{\pi}{2}}^{\frac{\pi}{2}} N_{i n t}(E, \theta) d \theta \times \eta_{i n j}(E) d E
$$

\section{S4. More information on hot electron energy collection and loss contribution}

The detailed optical and electrical models have been elucidated in the above section from $\mathrm{S} 1-\mathrm{S} 3$, where the processes about the energy collection and loss distribution in Figure $3 \mathrm{f}$ and Figure $4 \mathrm{f}$ can be obtained. For clarity, we show how the data of the collection and loss contribution can be calculated with the optoelectronic model in detail.

From the optical model, we get the optical absorption $(A)$ in the metal and reflection $(R)$ of the hot-electron device.

1) Considering the incident photon flux of $N_{\mathrm{ph}}$, the optical reflection loss $\left(N_{\mathrm{ph}-\text { ref }}\right)$ is:

$$
N_{\text {ph_ref }}=N_{\text {ph }} \times R
$$

2) The resistive dissipation loss $\left(N_{\text {ph_resis }}\right)$ is:

$$
N_{\text {ph_resis }}=N_{\text {ph }} \times A \times \eta_{\text {res }}
$$

where $\eta_{\text {res }}$ is the efficiency of resistive loss of the absorbed energy.

The hot-electron generation flux $\left(N_{\text {ph_excited }}\right)$ is:

$$
N_{\text {ph_excited }}=N_{\text {ph }} \times A \times \eta_{e h}
$$

where $\eta_{e h}$ is the efficiency of plasmonic decay into hot electrons.

3) The thermalization loss $\left(N_{\mathrm{ph}}\right.$ therm $)$ is the flux difference of hot electrons excited and reaching the interface:

$$
N_{\text {ph_therm }}=N_{\text {ph_excited }}-\int_{0}^{\infty} \int_{-\frac{\pi}{2}}^{\frac{\pi}{2}} N_{\text {int }}(E, \theta) d \theta d E
$$

where $N_{\text {int }}$ is the flux of electrons with excess energy $E$ to reach the interface under an angle $\theta$, as shown in the Eq. S7 in the section of S2.

4) The barrier loss $\left(N_{\mathrm{ph}}\right.$ barrier $)$ is the flux of hot electrons reaching the interface with excess energy $E<\varphi_{\mathrm{SB}}$ :

$$
N_{\text {ph_barrier }}=\int_{0}^{\varphi_{S B}} \int_{-\frac{\pi}{2}}^{\frac{\pi}{2}} N_{\text {int }}(E, \theta) d \theta d E
$$


5) The flux of collected hot electrons $\left(N_{\text {tot_succ }}\right)$ can be obtained from Eq. S18 in the supporting information:

$$
N_{\text {tot } \_ \text {succ }}=\int_{\varphi_{S B}}^{\infty} \int_{-\frac{\pi}{2}}^{\frac{\pi}{2}} N_{\text {int }}(E, \theta) d \theta \times \eta_{\text {inj }}(E) d E
$$

6) The momentum loss ( $\left.N_{\text {ph_momen }}\right)$ is the flux difference of hot electrons reaching the interface with excess energy $E>\varphi_{\mathrm{SB}}$ and collected:

$$
N_{\text {ph_momen }}=\int_{\varphi_{S B}}^{\infty} \int_{-\frac{\pi}{2}}^{\frac{\pi}{2}} N_{i n t}(E, \theta) d \theta d E-\int_{\varphi_{S B}}^{\infty} \int_{-\frac{\pi}{2}}^{\frac{\pi}{2}} N_{i n t}(E, \theta) d \theta \times \eta_{i n j}(E) d E
$$

Then each hot-electron collection and loss contribution can be obtained by dividing by $N_{\text {ph. }}$ The above are the details on how to calculate the hot-electron collection and loss contribution in Figure $3 \mathrm{f}$ and Figure 4f.

\section{$\underline{\text { 55. More information about hot electron relaxation mechanisms }}$}

The lifetimes $(\tau)$ of the three relaxation mechanisms under room temperature are shown in Table $\underline{\mathrm{S} 1}$.

Table S1. The lifetimes $(\tau)$ of the electron-electron (e-e) scattering, electron-phonon (e-ph) scattering, and phonon-phonon (ph-ph) thermalization.

\begin{tabular}{|c|c|c|c|}
\hline & e-e & e-ph & ph-ph \\
\hline$\tau$ & $40 \mathrm{fs}^{[\mathrm{S} 12, \mathrm{~S} 13]}$ & $800 \mathrm{fs}^{[\mathrm{S} 14, \mathrm{~S} 15]}$ & $86 \mathrm{ps}^{[\mathrm{S} 13]}$ \\
\hline
\end{tabular}

Below, we show these quantities as a function of temperature.

1. Electron-electron scattering: According to the Fermi liquid theory, ${ }^{[\mathrm{S} 16]}$ the quasiparticle $\underline{\text { lifetime of an electron with energy } \varepsilon \text { interacting with electrons having a Fermi distribution of }}$ temperature $T$ is,

$$
\frac{1}{\tau_{e-e}}=K \frac{\left(\pi k_{B} T\right)^{2}+\varepsilon^{2}}{1+\exp \left[-\varepsilon /\left(k_{B} T\right)\right]}
$$

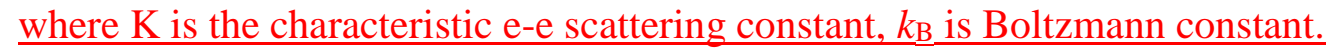

2. Electron-phonon scattering: The experimental data and the calculated average electronphonon energy relaxation time are shown in Figure S1. ${ }^{[\mathrm{S} 16]}$ 


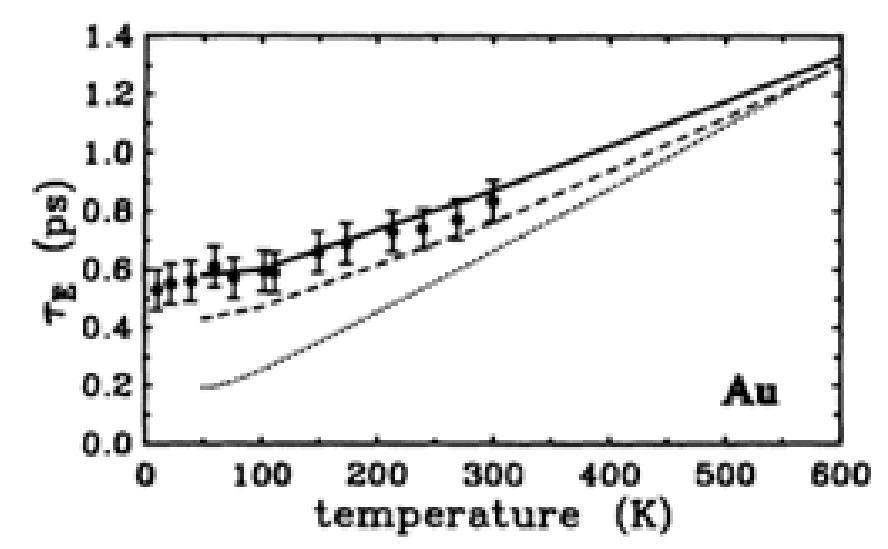

Figure S1. Comparison between experimental data and the calculated average electron-phonon energy relaxation time $\tau_{E}$ using a Boltzmann equation that accounts for both electron-electron and electron-phonon scattering. Electron-electron scattering rate: solid line, $K=0.1 \mathrm{fs}^{-2} \mathrm{eV}^{-1}$ : long-dashed line, $K=0.2 \mathrm{fs}^{-2} \mathrm{eV}^{-1}$; short-dashed line, $K=\infty$ (thermalized limit). For Ag and Au the electron-phonon coupling constant is $\mathrm{g}_{\infty}=3.5$ and $3.0 \times 10^{16} \mathrm{~W} \mathrm{~m}^{-3} \mathrm{~K}^{-1}$, respectively. Dots: experimental data.

3. Phonon-phonon scattering: The phonon relaxation times are shown in Figure S2. ${ }^{[\mathrm{S} 17]}$

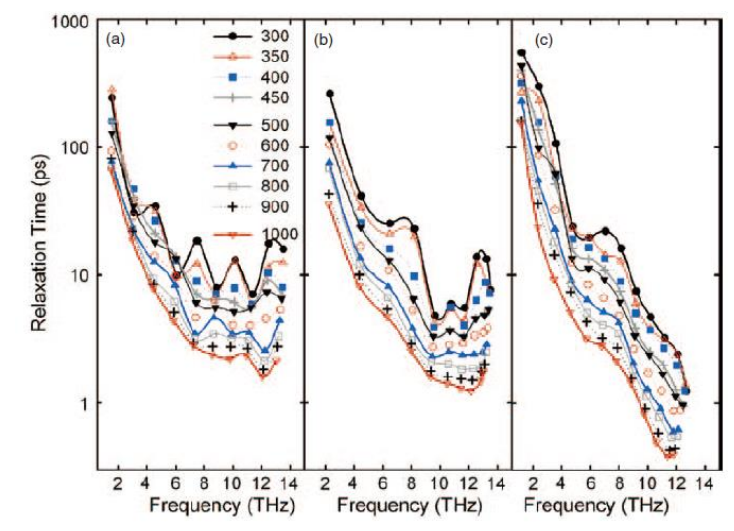

Figure S2. Longitudinal acoustic phonon relaxation times. Longitudinal acoustic phonon relaxation times extracted from normal mode decay times in the (a) $[1,0,0]$ (b) $[1,1,0]$ and (c) $[1,1,1]$ directions at ten different temperatures.

\section{References}

[S1] E. D. Palik, Handbook of Optical Constants of Solids, 1985.

[S2] Comsol Multiphysics; available from: http://www.comsol.com/ (accessed Jan 9, 2017).

[S3] X. Li, Y. Zhan. Appl. Phys. Lett. 102 (2013) 021101. 
[S4] A. M. Brown, R. Sundararaman, P. Narang, W. A. Goddard III, and H. A. Atwater, ACS Nano, 10 (2017) 957-966.

[S5] T. P. White and K. R. Catchpole, Appl. Phys. Lett. 101 (2012) 073905.

[S6] C. Ng, J. J. Cadusch, S. Dligatch, A. Roberts, T. J. Davis, P. Mulvaney, and D. E. Gómez, ACS Nano 2016, 10, 4704-4711.

[S7] T. Gong and J. N. Munday, Opt. Mater. Express 5 (2015) 2501-2512.

[S8] M. Kornbluth, A. Nitzan, and T. Seideman, J. Chem. Phys. 138 (2013) 174707.

[S9] D. A. Kovacs, J. Winter, S. Meyer, A. Wucher, and D. Diesing, Phys. Rev. B 76 (2007) 235408.

[S10] C. Scales and P. Berini, IEEE J. Quantum Electron. 46 (2010) 633-643.

[S11] H. Chalabi, D. Schoen, and M. L. Brongersma, Nano Lett. 14 (2014) 1374-1380.

[S12] F. Ladstädter, U. Hohenester, P. Puschnig, and C. Ambrosch-Draxl. Phys. Rev. B 2004, $\underline{70,235125 .}$

[S13] S. Link and M. A. El-Sayed. J. Phys. Chem. B 1999, 103, 8410.

[S14] N. Del Fatti, C. Flytzanis, and F. Vallee. Appl. Phys. B 1999, 68, 433.

[S15] R. Méjard, A. Verdy, M. Petit, A. Bouhelier, B. Cluzel, and O. Demichel. ACS Photonics $\underline{2016,3,1482 .}$

[S16] R. H. Groeneveld, R. Sprik, and A. Lagendijk. Phys. Rev. B 1995, 51, 11433.

[S17] A. S. Henry and G. Chen. J. Comput. Theor. Nanos. 2008, 5, 141. 